\title{
Polyethylene thickness is a risk factor for wear necessitating insert exchange
}

\author{
Bart G. Pijls • \\ Henrica M. J. Van der Linden-Van der Zwaag • \\ Rob G. H. H. Nelissen
}

Received: 24 August 2011 / Accepted: 1 November 2011 /Published online: 22 November 2011

(C) The Author(s) 2011. This article is published with open access at Springerlink.com

\begin{abstract}
Purpose The aim of this observational study was to investigate the optimal minimal polyethylene (PE) thickness in total knee arthroplasty (TKA) and identify other risk factors associated with revision of the insert due to wear. Methods A total of 84 TKA were followed for 11-16 years. All patients received the same prosthesis design (Interax; Howmedica/ Stryker) with halfbearings: separate PE-inserts medially and laterally. Statistical analysis comprised Coxregression to correct for confounding.

Results Eight knees (9.5\%) had been revised due to thinning inserts and an additional patient is scheduled for revision. PE thickness, diagnosis, BMI and weight are risk factors for insert exchange. For each millimetre decrease in PE thickness, the risk of insert exchange increases 3.0 times, which remains after correction for age, gender, weight, diagnosis and femoral-tibial angle. Insert exchange was 4.73 times more likely in OA-patients compared to RA-patients. For every unit increase in BMI and weight the risk for insert exchange increases 1.40 times and 1.14 times, respectively.

Conclusions In conclusion we therefore advise against the use of thin PE inserts in modular TKA and recommend PE inserts with a minimal 8-mm thickness.
\end{abstract}

\footnotetext{
B. G. Pijls $(\bowtie) \cdot H$. M. J. Van der Linden-Van der Zwaag •

R. G. H. H. Nelissen

Department of Orthopaedics, Leiden University Medical Center,

Albinusdreef 2, P.O. Box 9600, Postzone J-11-S, 2300 RC,

Leiden, The Netherlands

e-mail: b.g.c.w.pijls@lumc.nl

H. M. J. Van der Linden-Van der Zwaag

e-mail: h.m.j.van_der_linden@lumc.nl

R. G. H. H. Nelissen

e-mail: r.g.h.h.nelissen@lumc.nl
}

\section{Introduction}

Patients receiving a total knee arthroplasty (TKA) nowadays are heavier, more active, younger and have a longer life expectancy than those from previous decades [1]. Since these factors are associated with increased polyethelene (PE) wear, a higher number of revisions for wear may be expected $[2,3]$. For these reasons there is a growing interest in factors that are associated with increased wear [4].

From a biomechanical view, thin PE inserts have unfavourable wear characteristics [5]. This finding has been confirmed by clinical studies showing increased liner exchange for TKA with thin inserts compared to TKA with thick inserts $[6,7]$. These studies, however, do not take into account other factors that are associated with wear, e.g. weight, BMI, age and component position (confounders), and this causes concern for the validity of their results. Furthermore, the magnitude of the clinical effect is unclear: how much do thin inserts increase the risk of insert revision?

Since the thickness of the PE insert can be modified intraoperatively, the wear characteristics can be optimised by choosing an insert of a particular minimal thickness, thereby reducing the chance of wearing through and thus the chance for liner exchange in the long run. Controversy thus remains regarding the question of what this minimal thickness should be $[5,8]$.

The purpose of this observational study was (1) to investigate the optimal minimal PE thickness while correcting for confounders, (2) to identify other risk factors associated with revision of the insert due to wear and (3) to investigate the survival and clinical performance of the revised cases. 


\section{Patients and methods}

This was an observational study of a cohort of 84 TKA (57 patients), which had been followed for 11-16 years. All patients who received the Interax cruciate retaining TKA (Stryker / Howmedica) were included from an academic centre specialised in the treatment of rheumatoid arthritis. No other inclusion or exclusion criteria were applied. The study was approved by our institutional ethics committee (pp160/93) and patients gave informed consent [9].

A cohort design was chosen, because it reflects clinical reality by allowing the PE thickness to be optimised to the patient in order to assure proper stability and range of motion. Furthermore, randomisation is not considered appropriated, because of the risk of instability when a thin PE insert is allocated to a TKA with a too large flexion and extension gap [10]. Alternatively, randomisation could also result in the TKA being too tight in case a thick PE insert is allocated to a TKA with a too small flexion and extension gap [11].

There were 40 patients with rheumatoid arthritis (RA) (62 TKA), 16 patients with osteoarthritis (OA) (21 TKA), and one with sequelae after septic arthritis (one TKA). The mean age at operation was 67 years (range 35-87 years, SD 11.8 years). There were 45 women (66 TKA in women) and 12 men (18 TKA in men). All operations were performed between November 1993 and January 1998. At the last follow-up (11-16 years) there were 24 patients (28 TKA) alive and 33 (48 TKA) patients had died. For 32 of the deceased patients (47 TKA) it was known whether they had undergone a revision for insert exchange or not. One of the dead patients was lost to follow-up three years postoperatively because she had moved to a different region; she was dead at the time of the analysis. She received one TKA with 6-mm inserts and was considered to be revised in a worst-case analysis.

In order to rule out selection bias and confounding due to differences in prosthetic design only patients treated with the Interax TKA were included. With 84 TKA (168 inserts) available for follow-up of 11-16 years and a medium expected survival of these inserts of eight years [12], there was $90 \%$ power to detect a clinically relevant hazard ratio (Cox regression) of 2.0 [13] with a significance level of 0.05 , two-sided.

\section{Implant and inserts}

The Interax TKA features modular low conformity UHMWPE half bearings, which are separate bearings for the medial and lateral compartment, allowing a difference in PE thickness between the two compartments. In seven out of 84 TKA $(8 \%)$ there was a $2-\mathrm{mm}$ difference in PE thickness and the knees were stable. The minimal thickness of a halfbearing was $6 \mathrm{~mm}$ and sizes increased by $2-\mathrm{mm}$ increments. The PE inserts were sterilised by gamma radiation in air and machined from ram extruded GUR 415 resin containing calcium stearate. Two halfbearings for 84 TKA represent 168 halfbearings. There were 71 inserts of 6-mm thickness, 66 of 8$\mathrm{mm}, 18$ of $10-\mathrm{mm}$, nine of $12-\mathrm{mm}$ and four of 14-mm.

Follow-up routine and outcome measures

Preoperative leg alignment was determined on weightbearing long-leg radiographs. After surgery, patients were invited at predefined follow-up sessions at three weeks, six weeks, three months, six months and one year postoperatively and at yearly intervals thereafter for radiological and clinical evaluation according to the Knee Society system [14]. Revisions for PE wear (insert exchange) were recorded. Patients continued to be invited for follow-up after the insert exchange in order to evaluate the effectiveness of the revision procedure.

Since all patients were prospectively followed, the indication for insert exchange was defined as presence of increased thinning of at least one of the inserts during follow-up. The time to revision was set in order to prevent the metal femoral component coming into contact with the metal tibial tray (worn through insert). The tibial tray has medial and lateral metal side posts (see Fig. 1). These side posts fix the inserts, and if these are damaged, a full total knee revision is necessary.
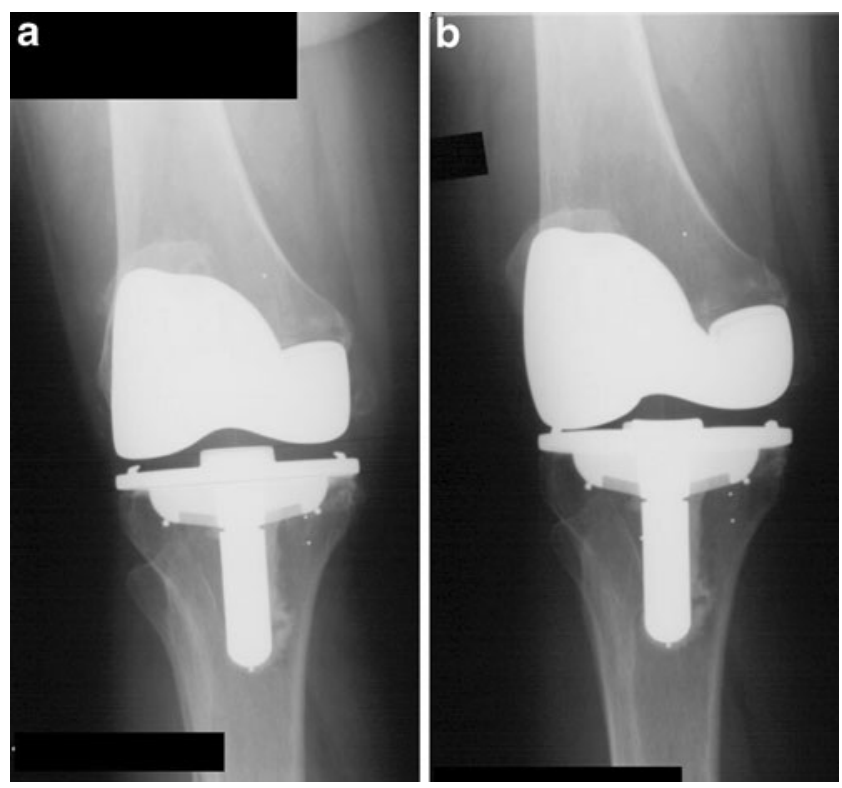

Fig. 1 Postoperative (a) and pre-revision (b) standing X-rays. The senior author used only the blinded pre-revision X-ray to determine which halfbearing required revision, i.e. the lateral halfbearing in this case. Also note the medial and lateral metal posts at the edge of the tibial plateau. At the pre-revision X-ray the lateral metal post is very close to the femoral component 
In order to reduce bias the senior author assessed blinded pre-revision radiographs to determine the halfbearing for which the insert exchange was indicated (medial, lateral or both; see Fig. 1). These results were crossed-checked with the surgical procedure records.

In order to determine the relative revision rate for 6-mm inserts compared to $8 \mathrm{~mm}$ or more, the cohort was divided into two groups: inserts of 6-mm thickness $(N=71)$ and inserts of $8 \mathrm{~mm}$ or more $(N=97)$. The baseline characteristics of both groups are presented in Table 1 .

\section{Statistical analysis}

BMI and weight $[3,15]$, age $[3,6]$ and alignment $[7,12]$ are associated with $\mathrm{PE}$ wear and could therefore confound the comparison of insert thickness. Additionally, activity level could be different between OA and RA patients which could also confound the comparison of insert thickness. Furthermore, difference in femoral and tibial component size is a specific reason for excessive wear for the Interax TKA, which is used in this study [12]. Cox regression has been used to take these factors and gender into account. Kaplan-Meier plots are used to visualise the survival free of insert revision. First, the influence of PE thickness on insert exchange rate is determined. Then, in subsequent analyses, the inserts are divided into two groups to evaluated the optimal thickness as proposed by Bartel et al. [5]: inserts of 6-mm thickness $(N=71)$ and inserts of $8-\mathrm{mm}$ or more thickness $(N=97)$.

\section{Results}

Indications for revision

The side of the most pronounced wear (medial, lateral or both) assessed during surgery corresponded in all cases with the indication made by the senior author on blinded radiographs.
Insert thickness and risk of insert exchange

There were eight (9.5\%) revisions for wear (liner exchange) at a mean 9.3 years postoperatively (range $3.1-14.7$ years): six for the lateral compartment and two for the medial compartment. Furthermore, one patient is scheduled to undergo insert exchange due to wear of the lateral insert at 15 years postoperatively.

PE thickness is associated with the risk of insert exchange. For each millimetre decrease in PE thickness, the risk of insert exchange increases 3.0 times HR3.0 (95\% CI 1.09-8.40).

Table 2 shows a significant 11 times higher revision risk for the $6-\mathrm{mm}$ inserts compared to the inserts of $8 \mathrm{~mm}$ or more; HR11 (95\%CI 1.4-93). This increased risk remains after correction for confounders. There is a 12 times higher revision risk for the 6-mm inserts compared to the $8-\mathrm{mm}$ inserts when the patient scheduled for revision is included in the analysis; HR12 (95\%CI 1.4-98).

The survival for the medial and lateral halfbearings is illustrated in Figs. 2 and 3. There is a significantly higher revision rate for the 6-mm lateral halfbearings compared to the lateral halfbearings of $8-\mathrm{mm}$ or more. This difference is not present in the medial compartment. However, since there are only two revisions for the medial compartment, this may not be enough to detect a difference in revision rate.

Assuming the patient lost to follow-up has undergone an insert exchange prior to being lost to follow-up - worst case analysis - there is a 13 times higher revision risk for the 6-mm inserts compared to the 8-mm inserts; OR 13 (95\%CI 1.7-109).

Risk factors for insert exchange

Risk factors for insert exchange, corrected for insert thickness, are presented in Table 3. Diagnosis, BMI and weight are significantly associated with the risk of insert exchange. Patient with OA have a 4.73 times higher risk for insert exchange than RA patients. For every point increase in BMI

Table 1 Baseline characteristics

$R A$ rheumatoid arthritis, $O A$ osteoarthritis, $S A$ sequelae after septic arthritis, KSS Knee Society system

Data are presented as mean, standard deviation (SD) and as counts. FTA angle is the femoral tibial angle measured on standing X-rays $(<175$ is valgus; $>175$ is varus)

\begin{tabular}{lll}
\hline Characteristics & 6 -mm inserts (SD) & $\begin{array}{l}\text { 8-mm or larger } \\
\text { inserts (SD) }\end{array}$ \\
\hline Number of inserts & 71 & 97 \\
Age (years) & $69(12.0)$ & $66(10.5)$ \\
Male / Female & $14 / 57$ & $22 / 75$ \\
Weight (kg) & $70(11.0)$ & $72(13.8)$ \\
BMI & $25.2(4.4)$ & $25.7(3.9)$ \\
RA / OA / SA & $59 / 12 / 0$ & $65 / 30 / 2$ \\
Medial / Lateral & $34 / 37$ & $50 / 47$ \\
Pre-operative FTA angle & $173(7.7)$ & $173(10.1)$ \\
Post-operative FTA angle & $176(2.4)$ & $177(3.0)$ \\
Pre-operative KSS score & $22(13)$ & $28(20)$ \\
Post-operative KSS score & $83(11)$ & $81(15)$ \\
\hline
\end{tabular}


Table 2 Risk of insert exchange. Crude and adjusted hazard ratios (HR) for insert exchange derived from the Cox-regression comparing 6-mm inserts to inserts of $8 \mathrm{~mm}$ or more

\begin{tabular}{lll}
\hline Parameter & $\begin{array}{l}\text { Hazard } \\
\text { ratio }\end{array}$ & $95 \%$ CI \\
\hline Crude & 11 & $1.4-93$ \\
Adjusted for: & & \\
Age & 11 & $1.4-94$ \\
Gender & 10 & $1.1-79$ \\
Weight $^{\text {a }}$ & 85 & $4.1-1758$ \\
BMI $^{\mathrm{a}}$ & 39 & $3-511$ \\
Diagnosis $_{\text {Medial / Lateral }}$ & 21 & $2.3-196$ \\
Post-operative FTA angle $^{\text {Post-operative KSS }}$ & 10 & $1.3-87$ \\
Difference in femoral and tibial & 15 & $1.7-143$ \\
component size [23] & 12 & $1.4-102$ \\
\hline
\end{tabular}

Note the 6-mm inserts have a significant 11 times higher revision risk compared to the inserts of $8 \mathrm{~mm}$ or more. This increased risk remains when adjusting for confounders

${ }^{\text {a }}$ When adjusting for weight or BMI the HR increases to 39 and 85, respectively, with a very wide confidence interval. This is due to the fact that BMI and weight are strong confounders and the cohort is relatively small with only eight insert exchanges

the risk for insert revision increases 1.40 times and for every kilogram gain in weight the risk increases 1.14 times.

Survival of the revised TKA

The mean follow-up after insert exchange for the eight revised TKA was 4.8 years (range $0.1-7.4$ years). There was one rerevision at three years after insert exchange for wear, instability

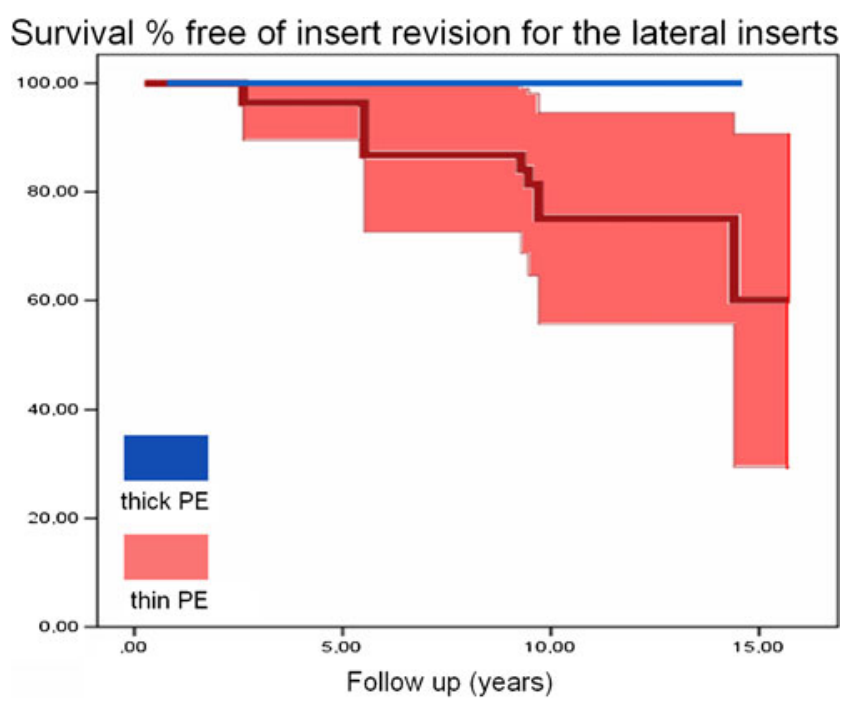

Fig. 2 Kaplan-Meier plot depicting the difference in survival free of insert revision between thin lateral and thick lateral inserts

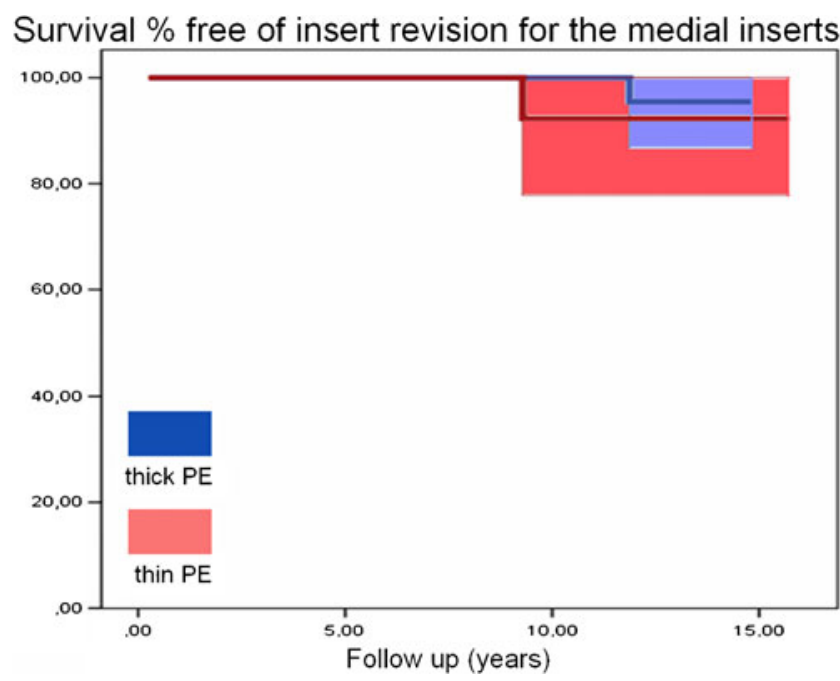

Fig. 3 Kaplan-Meier plot depicting the difference in survival free of insert revision between thin medial and thick medial inserts

and loosening of the femoral component. At the first revision (insert exchange only), this TKA received a low conformity PE insert from the original batch of the study (1993-1998). All other seven TKA had a mean KSS of 91 (SD 2.8) and have not been re-revised at last follow-up. They received PE inserts from 2002 and onwards at the time of revision, which are highly conforming, free of Ca-stearate and no longer sterilised by gamma radiation in air.

\section{Discussion}

The results indicate that PE thickness is indeed associated with the risk of insert exchange. In addition to PE thickness

Table 3 Risk factors for insert exchange adjusted for insert thickness

\begin{tabular}{|c|c|c|}
\hline Risk factors & $\begin{array}{l}\text { Hazard } \\
\text { ratio }^{a}\end{array}$ & $95 \% \mathrm{CI}$ \\
\hline Age & 1.04 & $0.97-1.11$ \\
\hline Weight & 1.14 & $1.04-1.25$ \\
\hline BMI & 1.40 & $1.14-1.74$ \\
\hline Diagnosis $^{\mathrm{b}}$ & 4.73 & $0.98-22.97$ \\
\hline KSS & 1.00 & $0.92-1.09$ \\
\hline Post-operative FTA angle & 1.30 & $0.90-1.86$ \\
\hline $\begin{array}{l}\text { Difference in femoral and tibial } \\
\text { component size [12] }\end{array}$ & 2.34 & $0.75-7.37$ \\
\hline
\end{tabular}

$C I$ confidence interval, BMI body mass index, KSS Knee Society system, FTA femoral tibial angle

Note: All insert exchanges were in females. It was therefore not possible to calculate a hazard ratio for gender

a All hazard ratios are adjusted for insert thickness

b Patients with osteoarthritis (OA) have a 4.73 times high risk for insert revision compared with rheumatoid arthritis (RA) patients 
diagnosis, BMI and weight also increase the risk of insert exchange. Apart from one case of instability with loosening of the femoral component, there were no re-revisions for the patients treated with insert exchange at a mean 4.8 years follow-up (range 0.1-7.4 years).

Insert exchange is the ultimate consequence of (linear) PE wear, which can be monitored on X-rays. With decreasing in vivo $\mathrm{PE}$ thickness the contact stress on the PE increases, which may lead to fatigue and subsequent fracture of the PE [5]. For this reason the risk of revision for wear could be higher in TKA with thin inserts. This effect may be amplified in modular TKA because modularity offers the advantage of exchanging only the PE insert and thus preserving the tibial and femoral component. If the ideal window for PE insert exchange is missed the tibial and femoral components could be damaged necessitating a full revision [16].

Although thickness of $\mathrm{PE}$ as a risk factor for revision for wear has been identified before [5-7], our study is the first to determine the magnitude of the clinical effect while correcting for known confounders (age, gender, weight, BMI, diagnosis, KSS score and alignment). Diagnosis, BMI and weight are also associated with an increased risk for insert exchange. OA patients have an almost five-fold higher risk for insert revision compared to RA patients. However, we should consider the fact that the RA patients, who are referred to our academic centre, are severe cases with involvement of multiple joints restricting their mobility. The decreased risk of insert exchange for RA patients may therefore be a result of decreased mobility rather than the result of RA alone [17]. Furthermore, the RA patients in this study are from an era in which aggressive treatment strategies with Disease-modifying Antirheumatic Drugs and biological response modifiers were being developed, so the results may not be fully translated to present day RA patients. In fact, the Australian National Joint Replacement Registry shows a smaller reduction in revision rates for RA [18] (0.55 revisions per 100 observed component years ) compared to OA (0.77 revision per 100 observed component years ); OA: $\mathrm{RA}=0.77: 0.55=1.4$ [95\%CI 1.14-1.70]. Therefore present day OA patients have a higher risk of insert exchange compared to RA which is likely to be in the magnitude of 1.4 rather than 5 .

For every point increase in BMI the risk for insert revision increases 1.40 times and for every kilogram gain in weight the risk increases 1.14 times. Whereas these risk factors have been described before, the magnitude of their clinical effect had not been previously determined [3].

Several authors have identified postoperative alignment in addition to difference in tibial and femoral component size to be risk factors for PE wear and subsequent insert revision [7, 12, 19]. Although we found indications for these risk factors-difference in component size HR 2.34 and FTA angle HR 1.30 (see Table 3)-there was no statistical significance. It is possible, however, that a cohort of 84 TKA is not large enough to detect them.

The results of the insert exchange cases are comparable to recently published larger patient series [20, 21]. Apart from one case of instability with loosening of the femoral component, there were no re-revisions for the patients treated with a insert exchange at a mean 4.8 years follow-up (range 0.1-7.4 years). The high revision rate for PE failure in the Interax TKA, used in our study, has been previously described by Sugimoto et al [12]. Sterilisation method (gamma radiation in air) in addition to implant design features are related to this high failure rate $[12,19]$. The large proportion of worn lateral inserts may be due to a combination of implant design features and the relatively large proportion of pre-operative valgus deformities in RA patients in both knee and hindfoot of those patients [12]. One case was lost to follow-up. When assuming that this patient had undergone a revision for $\mathrm{PE}$ wear in a different hospital - a worst case analysis - the increased risk of revision for the thin inserts compared to the thick inserts would even have been more substantial: 13 times increased versus 11 times increased. Therefore this case of lost to follow-up does not alter the conclusions.

An issue with PE thickness is the difference in actual minimal PE thickness and the size reported by the manufacturer. The real minimal PE thickness can be up to $4 \mathrm{~mm}$ thinner than the thickness reported by the manufacturer [22]. For this reason orthopaedic surgeons could still be using inserts of $6 \mathrm{~mm}$ or less without being aware of it. Regarding the inserts used in our study, we verified that minimal PE thickness of the Interax corresponds with the size reported by the manufacturer by measuring the minimal PE thickness with a calliper in a small sample of inserts.

A recent study has shown the catastrophic consequences of increased PE wear, leading to a high failure rate in a contemporary TKA design $[23,24]$. Therefore, the problems of excessive wear are not only a problem of the past $[25,26]$. Knowledge of risk factors for wear and the magnitude of their clinical effect is thus imperative. The recent GRADE guidelines are developed for grading the strength of evidence [13]. According to those guidelines a very strong association is defined as a relative risk of 5 or more. There should also be evidence of a dose response gradient and the association should remain after correction for confounders [13]. According to the GRADE guidelines, the evidence for PE thickness as a risk factor for wear and liner exchange may be classified as very strong, because the relative risk is greater than 5 (HR $=11$ ), there is evidence of a dose response gradient and the association remains after correcting for confounders [13]. Choosing the right minimal insert thickness is an elegant and cost-effective intraoperative measure to achieve a ten-fold reduction in the amount of revision operations for wear, which outweighs the required additional bone resection. In conclusion we therefore advise against the use of thin PE 
inserts in modular TKA and recommend PE inserts with a minimal 8-mm thickness.

Acknowledgements The authors would like to thank the Atlantic Innovation Fund (Atlantic Canada Opportunities Agency) for providing funding for this study. The Atlantic Innovation Fund did not take part in the design or conduct of the study; in the collection, management, analysis, or interpretation of the data; or in the preparation, review, or approval of the manuscript.

Open Access This article is distributed under the terms of the Creative Commons Attribution Noncommercial License which permits any noncommercial use, distribution, and reproduction in any medium, provided the original author(s) and source are credited.

\section{Reference}

1. Crowninshield RD, Rosenberg AG, Sporer SM (2006) Changing demographics of patients with total joint replacement. Clin Orthop Relat Res 443:266-272

2. Kurtz SM, Ong KL, Lau E, Widmer M, Maravic M, Gomez-Barrena E, de Fatima de Pina M, Manno V, Torre M, Walter WL, de Steiger R, Geesink RG, Peltola M, Roder C (2011) International survey of primary and revision total knee replacement. Int Orthop. Mar 15 [Epub ahead of print] doi:10.1007/s00264-00011-01235-00265

3. Tsao A, Mintz L, McRae CR, Stulberg SD, Wright T (1993) Failure of the porous-coated anatomic prosthesis in total knee arthroplasty due to severe polyethylene wear. J Bone Joint Surg Am 75(1):19-26

4. Fisher J, Jennings LM, Galvin AL, Jin ZM, Stone MH, Ingham E (2010) 2009 Knee Society Presidential Guest Lecture: Polyethylene wear in total knees. Clin Orthop Relat Res 468(1):12-18

5. Bartel DL, Bicknell VL, Wright TM (1986) The effect of conformity, thickness, and material on stresses in ultra-high molecular weight components for total joint replacement. J Bone Joint Surg Am 68(7):1041-1051

6. Bugbee WD, Ammeen DJ, Parks NL, Engh GA (1998) 4- to 10year results with the anatomic modular total knee. Clin Orthop Relat Res 348(348):158-165

7. Kilgus DJ, Moreland JR, Finerman GA, Funahashi TT, Tipton JS (1991) Catastrophic wear of tibial polyethylene inserts. Clin Orthop Relat Res 273(273):223-231

8. Meding JB, Ritter MA, Faris PM (2001) Total knee arthroplasty with $4.4 \mathrm{~mm}$ of tibial polyethylene: 10-year followup. Clin Orthop Relat Res 388:112-117

9. Nelissen RG, Valstar ER, Rozing PM (1998) The effect of hydroxyapatite on the micromotion of total knee prostheses. A prospective, randomized, double-blind study. J Bone Joint Surg Am 80(11):1665-1672

10. Higuchi H, Hatayama K, Shimizu M, Kobayashi A, Kobayashi T, Takagishi K (2009) Relationship between joint gap difference and range of motion in total knee arthroplasty: a prospective randomised study between different platforms. Int Orthop 33 (4):997-1000
11. Rousseau MA, Lazennec JY-Y, Catonne Y (2008) Early mechanical failure in total knee arthroplasty. Int Orthop 32(1):53-56

12. Sugimoto K, Iwai M, Okahashi K, Kaneko K, Tanaka M, Takakura Y (2005) Premature failure of the polyethylene tibial bearing surface of the Interax knee arthroplasty. J Arthroplasty 20 (5):658-663

13. Atkins D, Best D, Briss PA, Eccles M, Falck-Ytter Y, Flottorp S, Guyatt GH, Harbour RT, Haugh MC, Henry D, Hill S, Jaeschke R, Leng G, Liberati A, Magrini N, Mason J, Middleton P, Mrukowicz J, O'Connell D, Oxman AD, Phillips B, Schunemann HJ, Edejer TT, Varonen H, Vist GE, Williams JW Jr, Zaza S (2004) Grading quality of evidence and strength of recommendations. BMJ 328(7454):1490

14. Insall JN, Dorr LD, Scott RD, Scott WN (1989) Rationale of the Knee Society clinical rating system. Clin Orthop Relat Res 248:13-14

15. Spicer DD, Pomeroy DL, Badenhausen WE, Schaper LA, Curry JI, Suthers KE, Smith MW (2001) Body mass index as a predictor of outcome in total knee replacement. Int Orthop 25(14):246-249

16. Bert JM, Reuben J, Kelly F, Gross M, Elting J (1998) The incidence of modular tibial polyethylene insert exchange in total knee arthroplasty when polyethylene failure occurs. J Arthroplasty 13(6):609-614

17. Nouta KA, Pijls BG, Nelissen RG (2011) All-polyethylene tibial components in TKA in rheumatoid arthritis: a 25-year follow-up study. Int Orthop. Sep 1. [Epub ahead of print] doi:10.1007/ s00264-00011-01341-00264

18. Australian Orthopaedic Association National Joint Replacement Registry (2011) Annual Report 2010. http://wwwdmacadelaidee duau/aoanjrr/publicationsjsp?section=reports2010. Accessed 17 October 2011

19. Collier MB, Engh CA Jr, McAuley JP, Engh GA (2007) Factors associated with the loss of thickness of polyethylene tibial bearings after knee arthroplasty. J Bone Joint Surg Am 89 (6): 1306-1314

20. Griffin WL, Scott RD, Dalury DF, Mahoney OM, Chiavetta JB, Odum SM (2007) Modular insert exchange in knee arthroplasty for treatment of wear and osteolysis. Clin Orthop Relat Res 464:132-137

21. Willson SE, Munro ML, Sandwell JC, Ezzet KA, Colwell CW Jr (2010) Isolated tibial polyethylene insert exchange outcomes after total knee arthroplasty. Clin Orthop Relat Res 468(1):96-101

22. Chillag KJ, Barth E (1991) An analysis of polyethylene thickness in modular total knee components. Clin Orthop Relat Res 273:261-263

23. Swedish Knee Arthroplasty Registry (2009) Report 2009 V1.0. www.knee.nko.se. Accessed 06 September 2010

24. Reay E, Wu J, Holland J, Deehan D (2009) Premature failure of Kinemax Plus total knee replacements. J Bone Joint Surg Br 91 (5):604-611

25. Feng EL, Stulberg SD, Wixson RL (1994) Progressive subluxation and polyethylene wear in total knee replacements with flat articular surfaces. Clin Orthop Relat Res 299:60-71

26. Toksvig-Larsen S, Ryd L, Stentstrom A, Dansgard F, Jonsson K, Robertson O, Lindstrand A (1996) The porous-coated anatomic total knee experience. Special emphasis on complications and wear. J Arthroplasty 11(1):11-17 